\title{
Upper gastrointestinal bleeding in patients with end stage renal disease: causes, characteristics and factors associated with need for endoscopic therapeutic intervention
}

\author{
Syed Mudassir Laeeq, Abbas Ali Tasneem, Farina M. Hanif, \\ Nasir Hassan Luck, Rajesh Mandhwani, Rajesh Wadhva \\ Sindh Institute of Urology and Transplantation, Karachi, Sindh, Pakistan
}

\begin{abstract}
Background and Objectives: The risk of upper gastrointestinal bleeding (UGIB) is increased among the end-stage renal disease (ESRD) patients. The aim of the current study was to describe the causes and characteristics of UGIB in ESRD patients at our center and to assess the need for endoscopic therapeutic intervention (ETI) using Rockall (RS) and Glasgow Blatchford scores (GBS). Material and Methods: All patients with ESRD and UGIB with age $\geq 14$ years were included. Frequencies and percentages were computed for categorical variables. Chi square test or Fischer's exact test was used for statistical analysis. Results: A total of 59 subjects had a mean age of $47.25 \pm 15$ years. The most common endoscopic findings seen were erosions in $33(55.9 \%)$ patients, followed by ulcers in $18(30.3 \%)$ patients. ETI was required in $33(55.9 \%)$ patients, which included adrenaline injection in $19(32.3 \%)$, hemoclip in $9(15.2 \%)$ and argon plasma coagulation in $5(8.4 \%)$ patients. Factors associated with the need of ETI were identified as: a combined presentation of hematemesis and melena $(P=0.033)$, ulcer $(P=0.002)$ and associated chronic liver disease $(P=0.015)$. Six $(10.1 \%)$ patients died. Death was more common if ETI was not performed $(P=0.018)$. Conclusion: $E T I$ was more commonly required in patients on maintenance hemodialysis with UGIB, who had presence of combined hematemesis and melena, ulcers and associated chronic liver disease. A Glasgow Blatchford score of $>14$ was helpful in assessing the need for ETI in these patients.
\end{abstract}

Key words: Upper gastrointestinal bleed, End-stage renal disease, Rockall score, Glasgow Blatchford score

Address for Correspondence: Farina Hanif, Sindh Institute of Urology and Transplantation

Karachi, Sindh, Pakistan

Email: farinahanif@hotmail.com

\begin{tabular}{|l|}
\hline Access this article online \\
\hline $\begin{array}{l}\text { Website: } \\
\text { www.intern-med.com }\end{array}$ \\
\hline $\begin{array}{l}\text { DOI: } \\
\text { 10.1515/tim-2017-0019 }\end{array}$ \\
\hline Quick Response Code: \\
\\
\\
\\
\\
\\
\end{tabular}

\section{INTRODUCTION}

The incidence and prevalence of end-stage renal disease (ESRD) requiring hemodialysis imparts a substantial burden on the health care system worldwide. ${ }^{[1]}$ ESRD is associated with various pathological conditions including electrolyte imbalance, metabolic acidosis, malnutrition, bleeding disorders and various pathological conditions of gastrointestinal, cardiac, pulmonary and immunological system. ${ }^{[2]}$ A study conducted in Taiwan ${ }^{[1]}$ reported the incidence of upper gastrointestinal bleeding (UGIB) in ESRD patients to be 42.01 per 1000 person-years as compared to 22.7 in a study done at USA. ${ }^{[3]}$ Previous studies have not only shown the evidence of increased risk of UGIB in
ESRD patients in comparison to general population, but also its association with higher mortality. ${ }^{[1]}$ Gastroduodenal ulcers, esophagitis, vascular ectasia and gastric and duodenal erosions are among the frequently identified causative factors of UGIB in patients on maintenance hemodialysis. ${ }^{[2]}$ Various mechanisms have been proposed leading to gastrointestinal (GI) bleed in this population including uremia-induced platelet dysfunction and increased risk of vascular malformation. ${ }^{[2]}$

Since GI diseases are common in ESRD patients, multiple studies have been conducted to study the endoscopic findings in this population. Most common reason for endoscopic evaluation has been UGIB. ${ }^{[2,4]}$ Several scoring systems have also been 
developed to classify patients with UGIB according to their outcome. One such scoring system is the Glasgow Blatchford bleeding Score (GBS), a well-established tool to stratify patients in dire need of intervention from UGIB on the basis of their history, physical examinations and laboratory findings ${ }^{[5]}$ Another is the pre-endoscopic Rockall score (PERS), ${ }^{[6]}$ which uses clinical criteria (increasing age, co-morbidity and shock) along with endoscopic findings. This score helps identify patients at risk of a poor outcome following UGIB. These scores are used in the general population but have never been used in ESRD patients.

Although various studies have been performed worldwide to identify the cause of UGIB in the general population, little work has been done to identify its causes in patients with ESRD. Besides, not much work has been done to identify the factors associated with the need for endoscopic therapeutic intervention (ETI) to control such bleeding. The aim of this study was, therefore, to study the causes and characteristics of UGIB in ESRD patients and to identify the factors associated with the need for ETI. The other objective was to assess the usefulness of using Rockall (RS) and GBS in ESRD patients.

\section{MATERIAL AND METHODS}

This prospective study was conducted in a tertiary care hospital. The study was performed over a period of 6 months. The proforma designed for this study included questions about the patients' demographic details, laboratory reports and clinical/physical findings. Using this information, PERS and GBS were calculated for each patient. The GBS was calculated from the values of the following parameters: clinical (systolic blood pressure, heart rate, melena and/or syncope, hepatic disease and/or cardiac failure) and laboratory (blood urea nitrogen and hemoglobin) parameters. ${ }^{[5]}$ PERS was calculated from the values of the following parameters: age, shock, and comorbidity. ${ }^{[6]}$ All patients underwent upper GI endoscopy (GIF 150 Olympus gastroscope) under conscious sedation. Methods to achieve hemostasis, if needed, were recorded in proforma.

All patients aged 14 years and older having ESRD and presenting with UGIB were included in this study, while patients not eligible for endoscopy were excluded. Questions were asked and the questionnaires were filled out personally by the principal investigator himself. An informed consent was obtained prior to this. Data entry and analysis was done using Statistical Program for Social Sciences (SPSS) version 20.0 (IBM Corporation, Armonk, NY, USA). Results were presented as means \pm standard deviation (SD) for quantitative data or as numbers with percentages for qualitative data. Statistical differences in quantitative data were determined using t-test or Mann-
Whitney U test. The Fisher's exact test or chi-square test was used for qualitative data. Area under curve (AUC) analysis was utilized to determine cut off value of GBS for prediction of ETI. A $P$ value $<0.05$ was considered statistically significant.

\section{RESULTS}

A total of 59 patients with UGIB and ESRD who presented in endoscopy unit of SIUT during this period were included in our study. Of these, $42(71.2 \%)$ were males and $17(28.8 \%)$ females. Mean age was $47.25 \pm 15.0$ years (range: $14-80$ years). Most of the patients $(74.6 \%)$ were below 60 years of age, while 15 (25.4\%) were above this age limit. The mean systolic blood pressure was 99.6 $\pm 17.96 \mathrm{mmHg}$ (range: $78-140 \mathrm{mmHg}$ ). The mean pulse was $107 \pm 14.9$ beats per minute (range: $88-154$ beats per minute). Fifty-seven (69.6\%) patients presented with melena; of which, $39(66.1 \%)$ had melena alone, while 18 $(30.5 \%)$ had both hematemesis and melena. One patient $(1.7 \%)$ had hematemesis only and four $(6.78 \%)$ patients also developed syncope.

The most common cause of UGIB identified in our study was erosions, which were seen in $33(55.9 \%)$ patients, followed by ulcers in 18 (30.3\%), telangiectasia in $5(8.5 \%)$ and Dieulafoy's lesions in $3(5.1 \%)$ patients. The most common location of bleeding in our study was found to be the duodenum seen in $44(74.6 \%)$ patients, followed by the stomach in $15(25.4 \%)$ patients. Erosions were most commonly found in the duodenal bulb and the junction of first and second part of duodenum in 31 patients, followed by gastric antrum in 2 patients. Ulcers too were most commonly found in duodenal bulb (12 patients) followed by 3 at gastroduodenal junction, 2 in antrum and 1 in distal duodenum. Telangiectasias were located in the antrum and body of stomach. Dieulafoy's lesions were seen in the proximal stomach (lesser curvature).

Thirty-seven $(62.7 \%)$ patients had UGIB secondary to lesions that were classified as Forrest class equal to or less than II, while $22(37.3 \%)$ patients had class III lesions. Among those who had lesions of class I type: spurting from a bleeding vessel (Forrest Ia) was seen in $4(6.8 \%)$, while oozing of blood (Forrest Ib) was seen in 15 (25.4\%) patients. A visible vessel (Forrest IIa) and adherent clot (Forrest IIb) was seen in $4(6.8 \%)$ while hematin on ulcer base (Forrest IIc) was seen in $10(16.9 \%)$ patients. Endoscopic finding of a clean based ulcer (Forrest III) was noted in $22(37.3 \%)$ cases.

To achieve hemostasis, various endoscopic therapeutic modalities were employed. Of the 33 cases of UGIB from erosions, injection adrenaline was employed in 10 (30.3\%) 
patients, while the remaining were managed conservatively with pharmacological intervention. Of the 18 cases of UGIB secondary to ulcers, 9 patients were treated with injection adrenaline, 6 patients were treated with a combination of injection adrenaline and hemoclip application, while 3 patients had healing phase ulcers and were managed with pharmacological measures only. All the five cases of telangiectasias were treated with argon plasma coagulation (APC), while all cases with Dieulafoy's lesions were managed with hemoclip application. In our study, the patients with ESRD that were treated for UGIB, $6(10.16 \%)$ patients died. Statistical analysis showed that death was more likely to occur if the ETI was not performed $(P=0.041)$.

The sensitivity and specificity of GBS for predicting ETI in UGIB patients, at cut off of 14 , was $81.2 \%$ and $42.3 \%$, respectively, with an AUC of 0.63 (Figure 1). To identify the factors that predict the need of ETI in hemodialysis patients presenting with UGIB, univariate and multivariate analysis was performed. Forrest classification was omitted from analysis as the need for ETI is obvious for class II and less. On univariate analysis, factors that were found to be associated with the need for ETI included patient age less than $60(P=0.041)$, presentation with hematemesis and melena $(P<0.001)$, heart rate $>100$ $(P=0.035)$, ulcer as the cause of UGIB $(P<0.001)$, gastric location of UGIB $(P<0.001)$, presence of liver cirrhosis

\begin{tabular}{|c|c|c|c|c|}
\hline \multirow[t]{2}{*}{ Clinical Parameter } & & \multirow[t]{2}{*}{$N$} & \multirow{2}{*}{$\begin{array}{l}\text { ETI performed } \\
N(\%)\end{array}$} & \multirow[t]{2}{*}{$P$ value } \\
\hline & & & & \\
\hline \multirow[t]{2}{*}{ Age } & $<60$ & 44 & $28(63.6)$ & 0.041 \\
\hline & $>60$ & 15 & $5(33.3)$ & \\
\hline \multirow[t]{2}{*}{ Gender } & Male & 42 & $22(52.3)$ & 0.563 \\
\hline & Female & 17 & $11(64.7)$ & \\
\hline \multirow[t]{2}{*}{ Presentation } & Hematemesis \& Melena & 19 & 15 (78.9) & 0.011 \\
\hline & Melena alone & 57 & $31(54.3)$ & \\
\hline \multirow[t]{2}{*}{ Heart rate } & $<100$ & 25 & $10(40)$ & 0.035 \\
\hline & $>100$ & 34 & $23(67.6)$ & \\
\hline \multirow[t]{2}{*}{ Liver Cirrhosis } & Present & 6 & $6(100)$ & 0.022 \\
\hline & Absent & 53 & 27 (50.9) & \\
\hline \multirow[t]{2}{*}{ Cause of UGIB } & Ulcers & 18 & $15(83.3)$ & 0.005 \\
\hline & Non-ulcer bleed & 41 & 18 (43.9) & \\
\hline \multirow[t]{2}{*}{ Location of UGIB } & Stomach & 15 & $12(80)$ & 0.038 \\
\hline & Duodenum & 44 & $21(47.7)$ & \\
\hline \multirow[t]{2}{*}{ PERS } & Less than 5 & 51 & $30(58.8)$ & 0.259 \\
\hline & More than 5 & 8 & $3(37.5)$ & \\
\hline \multirow[t]{2}{*}{ GBS* } & Less than 14 & 17 & $6(35.2)$ & 0.042 \\
\hline & More than 14 & 42 & $27(64.2)$ & \\
\hline
\end{tabular}

GBS: Glasgow Blatchford bleeding score; PERS: Pre-endoscopic Rockall score; UGIB: upper gastrointestinal bleed.

${ }^{*}$ Cut off was derived from receiver operating curve significant $P$ value $<0.05$
$(P=0.022)$ and GBS of $>14(P<0.001)$ (Table 1$).$ However, on multivariate analysis, statistically significant association of ETI was observed with a presentation with combined hematemesis and melena $(P=0.033)$, ulcer as a cause of $\operatorname{UGIB}(P=0.002)$ and presence of chronic liver disease $(P=0.015)$ (Table 2).

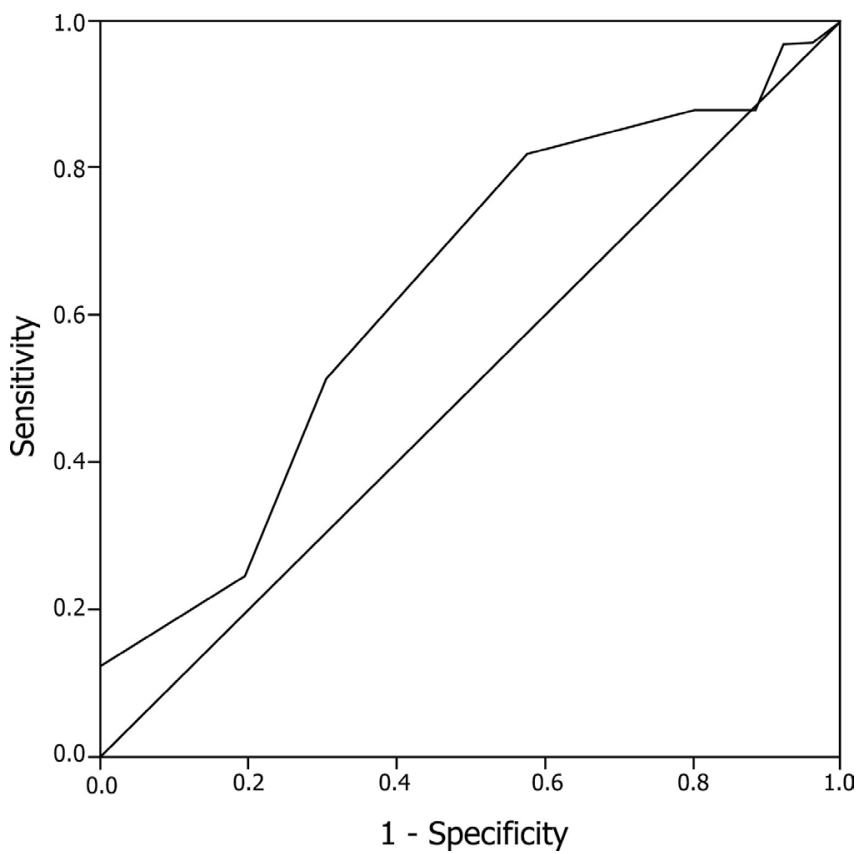

Figure 1: ROC curve of the sensitivity and specificity of GBS for predicting ETI in UGIB patients. 
Laeeq et al.: Upper gastrointestinal bleeding in patients with end stage renal disease

\begin{tabular}{|c|c|c|c|c|c|}
\hline \multirow[t]{2}{*}{ Model } & \multicolumn{2}{|c|}{ Unstandardized Coefficients } & \multirow{2}{*}{$\begin{array}{l}\text { Standardized } \\
\text { coefficients } \\
\text { Beta }\end{array}$} & \multirow[t]{2}{*}{$\boldsymbol{t}$} & \multirow[t]{2}{*}{$P$ value } \\
\hline & B & Std Error & & & \\
\hline Age & -0.053 & 0.141 & -0.047 & -0.376 & 0.709 \\
\hline Heart rate & -0.110 & 0.124 & -0.110 & -0.889 & 0.378 \\
\hline Presentation & 0.274 & 0.125 & 0.258 & 2.195 & 0.033 \\
\hline Location & 0.220 & 0.136 & 0.193 & 1.616 & 0.112 \\
\hline Ulcer & 0.400 & 0.123 & 0.371 & 3.251 & 0.002 \\
\hline Liver Cirrhosis & 0.508 & 0.201 & 0.309 & 2.523 & 0.015 \\
\hline GBS & 0.139 & 0.125 & 0.127 & 1.113 & 0.271 \\
\hline
\end{tabular}

GBS: Glasgow Blatchford bleeding score; significant $P$ value $<0.05$

\section{DISCUSSION}

Gastrointestinal bleeding has been frequently reported as a complication of advanced chronic renal failure ${ }^{[1-3]}$ and is the cause of mortality in 3-7\% of such patients. ${ }^{[1]}$ Multiple factors have been proposed that increase the risk of UGIB in ESRD patients including platelet dysfunction due to uremia, high prevalence of arteriovenous malformations (AVM), various co-morbidities like cardiovascular disease, diabetes mellitus, liver cirrhosis and old age. ${ }^{[4,7-8]}$ Anemia has also been suggested to increase the risk of bleeding as red blood cells assist in interface of platelets at the site of injury. The improvement of bleeding time in uremic patients with erythropoietin and blood transfusions also favors this theory. ${ }^{\left[{ }^{[8]}\right.}$ The exposure to medications that affects the clotting cascade like anticoagulants, antiplatelets and non-steroidal anti-inflammatory agents (NSAIDS) also place ESRD population at increased risk of UGIB. ${ }^{[4,9]}$

Past studies have demonstrated that in ESRD patients, bleeding from the upper GI tract is more common than from the lower GI tract. ${ }^{[10]}$ Also, gastroduodenal ulcers and erosions are among the frequent causative factors for UGIB in hemodialysis patients. ${ }^{[11,12]}$ Our study demonstrated erosions as the commonest cause of UGIB in the ESRD population, mostly involving the duodenum (93.9\%). A study by Sotoudehmanesh $e t$ al. ${ }^{[13]}$ revealed that duodenal erosions were the commonly seen findings in EGD, while other studies demonstrated gastric erosions in patients on maintenance hemodialysis. ${ }^{[11,12]}$

In our study, peptic ulcers were the second most frequent cause of UGIB. This finding was similar to the study by Armando et al. , $^{[12]}$ which demonstrated that duodenal ulcers and gastric ulcers were commonly observed in ESRD patients. In our study, UGIB secondary to ulceration was diagnosed in $18(30.5 \%)$ patients. For patients with spurting vessels, hemostasis was achieved with a combination of hemoclip and adrenaline injection in $6(33.3 \%)$ patients, while for ulcers with oozing of blood only, injection adrenaline alone was used in $9(50 \%)$ patients. No intervention was performed in $3(16.7 \%)$ patients. The fact that combination therapy is more effective was also seen in a study by Chung et al., ${ }^{[15]}$ who demonstrated that patients having UGIB secondary to an ulcer that was spurting blood, dual therapy with epinephrine injection and thermal therapy, produced better outcomes compared to the injection therapy alone.

Although the commonest presentation of UGIB in our study population was melena, ETI was more commonly required in those who had hematemesis too. Similarly, although duodenum was the commonest site of bleeding, ETI was more commonly required when the source was stomach. Also, erosions were more commonly found in such patients, but ETI was required with ulcers. The common causes of non-variceal UGIB required only acid suppressive therapies and endoscopic hemostasis was rarely required. ${ }^{[14]}$ Likewise, in our study, hemostasis with injection epinephrine was required in only 10 patients out of 33 patients diagnosed with erosions on endoscopy.

Since UGIB is associated with high mortality, scoring systems have been developed to predict the outcome of therapy and need of endoscopic therapeutic intervention (ETI). The Rockall score, is one such scoring system that has parameters that include pre-endoscopic (age, shock and comorbidity) and post-endoscopic (diagnosis and presence or absence of endoscopic stigmata of recent hemorrhage) factors. ${ }^{[6]}$ Various studies have demonstrated high mortality with higher Rockal scores. ${ }^{[16]}$ In our study, we calculated the pre-endoscopic Rockall score (PERS) and scores greater than 5 were associated with high mortality, although this was not shown to be statistically significant $(P=0.259)$. The Glasgow Blatchford score is another scoring system which also uses clinical and laboratory parameters. ${ }^{[5]}$ Various studies performed in the past have shown that GBS lacks high specificity in stratifying patients into high and low risk groups. ${ }^{[17,18]}$ 
In our study, at a threshold of $14, \mathrm{GBS}$ had a sensitivity of $81.2 \%$ and specificity of 42.3 with AUC of 0.63 (Figure 1 ). The threshold of 14 is high as compared to the externally validated studies. ${ }^{[17,19]}$ The specificity decreased even further with a rising GBS score. This discrepancy in results can be due to the difference in study population. Our study population consisted solely of ESRD patients who were usually anemic, had high blood urea level and had associated cardiac disease. On univariate analysis, GBS showed statistical significance but this association could not be demonstrated on multivariate analysis.

Not much research has been done worldwide in the past where factors associated with need for ETI in UGIB patients with ESRD have been evaluated. The factors we found in our study to be associated with need for ETI were patient age less than 60 years, presentation with hematemesis and melena, heart rate greater than 100 at the time of UGIB, gastric and melena source of bleeding, presence of chronic liver disease, presence of ulcer as the cause of UGIB and a GBS score of greater than 14. Our study showed that younger patients with UGIB required ETI more frequently than the elderly. This can be explained by the fact that ESRD patients in subcontinent are younger than western population. ${ }^{[20]}$ This is also demonstrated in our study, where the majority of patients were younger than 60 years with mean age of $47.25 \pm 15$ years.

Also, chronic liver disease patients were found to have higher chances of requiring ETI. This can again be explained by the fact that bleeding in such cases is more common due to coagulopathy secondary to the combined effect of both renal and liver disease. Therefore, it is important to note that the chances of requiring endoscopic management for UGIB in ESRD patients are higher if the patient presents with hematemesis and has a chronic liver disease.

To our knowledge it is the first prospective observational study which used scoring system in ESRD population. Our study also has some limitations to assess the need of ETI in UGIB: it is a single center study with small sample size of 59 patients. Furthermore, all ESRD patients were hemodialysed as in our center peritoneal dialysis was not frequently performed.

\section{CONCLUSION}

In conclusion, the most common presentation of UGIB in patients with ESRD was melena, the most common cause was erosions and the most common site was duodenum. However, ETI was more commonly required in patients who presented with both hematemesis and melena, whose cause of UGIB was ulcers and site of bleeding was stomach. A Glasgow Blatchford score of greater than 14 was helpful in assessing the need for ETI in patients on maintenance hemodialysis with UGIB.

\section{Conflict of Interest}

The authors have no conflicts of interest to declare.

\section{REFERENCES}

1. Kuo Kuo CC, Kuo HW, Lee IM, Lee CT, Yang CY. The risk of upper gastrointestinal bleeding in patients treated with hemodialysis: a populationbased cohort study. BMC Nephrol 2013;14:15.

2. Spinelli G, Tomasello G, Damiani F, Damiani P, Monte AI. Endoscopic findings in chronic renal failure: review of literature. Acta Medica Mediterranea. 2012;28:261-5.

3. Wasse H, Gillen DL, Ball AM, Kestenbaum BR, Seliger SL, Sherrard D. et al. Risk factors for upper gastrointestinal bleeding among end-stage renal disease patients. Kidney Int 2003;64:1455-61.

4. Kalman RS, Pedrosa MC. Evidence-based review of gastrointestinal bleeding in the chronic kidney disease patient. Semin Dial 2015;28:68-74.

5. Blatchford O, Murray WR, Blatchford M. A risk score to predict need for treatment for upper-gastrointestinal haemorrhage. Lancet 2000;356:131821.

6. Rockall TA, Logan RF, Devlin HB, Northfield TC. Risk assessment after acute upper gastrointestinal haemorrhage. Gut 1996;38:316-21.

7. Liang CC, Wang SM, Kuo HL, Chang CT, Liu JH, Lin HH. et al. Upper gastrointestinal bleeding in patients with CKD. Clin J Am Soc Nephrol 2014;9:1354-9.

8. Chalasani N, Cotsonis G, Wilcox CM. Upper gastrointestinal bleeding in patients with chronic renal failure: role of vascular ectasia. Am J Gastroenterol 1996;91:2329-32.

9. Yang JY, Lee TC, Montez-Rath ME, Paik J, Chertow GM, Desai M, Winkelmayer WC. Trends in acute nonvariceal upper gastrointestinal bleeding in dialysis patients. J Am Soc Nephrol 2012;23:495-506.

10. Gheissari A, Rajyaguru V, Kumashiro R, Matsumoto T. Gastrointestinal hemorrhage in end stage renal disease patients. Int Surg 1990;75:93-5.

11. Ikeda N, Inoue T, Kobayashi K, Watanabe Y, Suzuki H. Emergency Gastrointestinal Bleeding in Dialysis Patients: Five Years' Experience in a Single Dialysis Center. Urol Nephrol Open Access J 2015;2:00021

12. Negri AL, Kido N, Estraviz HO, Morelli OH Jr, Morelli OH. Upper gastrointestinal bleeding in patients in chronic hemodialysis. Nephron 1994;67:130.

13. Sotoudehmanesh R, Ali Asgari A, Ansari R, Nouraie M. Endoscopic findings in end-stage renal disease. Endoscopy 2003;35:502-5.

14. Gralnek IM, Dumonceau JM, Kuipers EJ, Lanas A, Sanders DS, Kurien $\mathrm{M}$, Rotondano G, et al. Diagnosis and management of nonvariceal upper gastrointestinal hemorrhage: European Society of Gastrointestinal Endoscopy (ESGE) Guideline. Endoscopy 2015;47:a1-46.

15. Chung SS, Lau JY, Sung JJ, Chan AC, Lai CW, Ng EK, et al. Randomised comparison between adrenaline injection alone and adrenaline injection plus heat probe treatment for actively bleeding ulcers. BMJ 1997;314:130711.

16. Lin SC, Wu KL, Chiu KW, Lee CT, Chiu YC, Chou YP, et al. Risk factors influencing the outcome of peptic ulcer bleeding in end stage renal diseases after initial endoscopic haemostasis. Int J Clin Pract 2012;66:774-81.

17. Pang SH, Ching JY, Lau JY, Sung JJ, Graham DY, Chan FK. Comparing the Blatchford and pre-endoscopic Rockall score in predicting the need for endoscopic therapy in patients with upper GI hemorrhage. Gastrointest Endosc 2010;71: 1134-40.

18. Masaoka T, Suzuki H, Hori S, Aikawa N, Hibi T. Blatchford scoring system is a useful scoring system for detecting patients with upper gastrointestinal bleeding who do not need endoscopic intervention. J Gastroenterol Hepatol 2007;22:1404-8. 
19. Stanley AJ, Ashley D, Dalton HR, Mowat C, Gaya DR, Thompson E. et al. Outpatient management of patients with low-risk upper-gastrointestinal haemorrhage: multicentre validation and prospective evaluation. Lancet 2009;373:42-7.

20. Sakhuja V, Kohli HS. End-stage renal disease in India and Pakistan: incidence, causes, and management. Ethn Dis 2006;16:S2.
How to cite this article: Laeeq SM, Tasneem AA, Hanif FM, Luck NH, Mandhwani R, Wadhva R. Upper gastrointestinal bleeding in patients with end stage renal disease: causes, characteristics and factors associated with need for endoscopic therapeutic intervention. J Transl Intern Med 2017; 5: 106-111. 